Radial and Nonradial Pulsations as Probes of Stellar Physics

ASP Conference Series, Vol. 259, 2002

C. Aerts, T.R. Bedding, \& J. Christensen-Dalsgaard, eds.

\title{
The Contribution of GEOS to the Study of Stellar Pulsations
}

\author{
R. Boninsegna, J. Vandenbroere, and the GEOS team \\ Groupe Européen d'Observation Stellaire, 23 Parc de Levesville, 28300 \\ Bailleau l'Evêque, France \\ J.F. Le Borgne \\ GEOS and Laboratoire d'Astrophysique, Observatoire Midi-Pyrénées, 14 \\ Avenue E. Belin, 31400 Toulouse, France
}

\section{Introduction}

The GEOS, Groupe Européen d'Observation Stellaires, is composed of observers living in France, Italy, Spain, Belgium and Switzerland. The main purpose is to give amateur astronomers the opportunity of carrying out scientific analyses in specific fields. Further details can be found at the web site

\section{http://www.upv.es/geos/}

In the past years, GEOS has approached the analysis of visual estimates of variable stars (Ralincourt et al., 1987) in an original manner, obtaining accurate light curves on red semiregulars. Moreover, the collaboration with teams of professional researchers allowed the group to obtain interesting results on the double--mode Cepheid EW Sct (Figer et al., 1991), on the Be star OT Gem (Arellano Ferro et al., 1998) and on the eclipsing binary V753 Cyg (Beltraminelli et al., 2000). More recently, several GEOS members have started collecting a large sample of times of maximum brightness of RR Lyr stars in order to build-up a database as extensive as possible. Such a database is decribed below together with the results of a campaign on $R R$ Lyr itself.

\section{The GEOS RR Lyr database}

The GEOS RR Lyr database is a free-access web tool whose aim is to collect data on field RRab and RRc stars and to make these data available. At present, it contains close to 30,000 times of maximum on 1,700 different stars. The RR Lyrae GEOS database is freely available and is hosted by the Laboratoire d'Astrophysique of Observatoire Midi-Pyrénées at the address:

\section{http://webast.ast.obs-mip.fr/people/leborgne/dbRR/}

The data date back to the end of XIXth century, when the first RR Lyr stars were discovered. RR Lyr stars can display period changes (see Vandenbroere \& Berthold, 2001 for the behaviour of UZ CVn) and our database can be used to monitor them, as the reason for these changes is still unknown. Apart from this secular period variations, RR Lyr stars exhibit another not fully explained phenomenon: the Blazkho effect, named after the Russian astronomer who discovered it in his observations of RW Dra in 1907. This periodic modulation of 


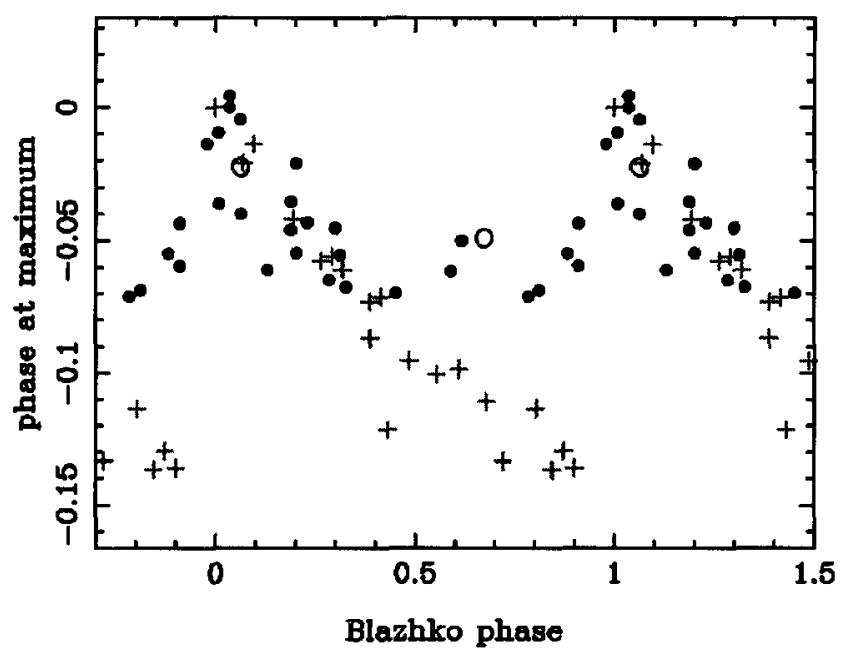

Figure 1. The Blazhko effect of RR Lyr. Crosses indicate Preston et al.'s (1965) observations, while the new GEOS observations are indicated by open circles (CCD) and filled circles (visual times of maxima).

the RR Lyr pulsation (periods from 20 to 200 days) may also be studied with the aid of our database. The preliminary results on a multitechnique campaign (June 2000) on RR Lyr itself are shown in Fig. 1; the Blazhko phase is calculated with respect to $P=40.812289 \mathrm{~d}$ (Preston et al., 1965).

\section{References}

Arellano-Ferro, A., Sareyan, J.P., Avila, J.J., Gonzales, F., Dumont, M., \& the GEOS team 1998, A\&AS, 127, 455

Beltraminelli, N. \& the GEOS team 2000, in ASP Conf. Series, Vol. 220, Amateur - Professional Partnerships in Astronomy, eds. J.R. Percy \& J.B. Wilson (San Francisco ASP), 75

Figer, A., Poretti, E., Sterken, C., \& Walker, N. 1991, MNRAS, 249, 563

Preston, G.W., Smak, J., \& Paczynski, B. 1965, ApJS, 12, 104

Ralincourt, Ph., Poretti E., \& Boistel, G. 1987, Ap\&SS, 134, 135

Vandenbroere, J. \& Berthold, T., 2001, IBVS, 5170 